\title{
Indentation-Induced Mechanical Deformation Behaviors of AlN Thin Films Deposited on c-Plane Sapphire
}

\author{
Sheng-Rui Jian' ${ }^{1}$ and Jenh-Yih Juang ${ }^{2}$ \\ ${ }^{1}$ Department of Materials Science and Engineering, I-Shou University, Kaohsiung 84001, Taiwan \\ ${ }^{2}$ Department of Electrophysics, National Chiao Tung University, Hsinchu 300, Taiwan
}

Correspondence should be addressed to Sheng-Rui Jian, srjian@gmail.com

Received 16 November 2011; Accepted 13 January 2012

Academic Editor: Edward Andrew Payzant

Copyright ( $\odot 2012$ S.-R. Jian and J.-Y. Juang. This is an open access article distributed under the Creative Commons Attribution License, which permits unrestricted use, distribution, and reproduction in any medium, provided the original work is properly cited.

\begin{abstract}
The mechanical properties and deformation behaviors of AlN thin films deposited on $c$-plane sapphire substrates by helicon sputtering method were determined using the Berkovich nanoindentation and cross-sectional transmission electron microscopy (XTEM). The load-displacement curves show the "pop-ins" phenomena during nanoindentation loading, indicative of the formation of slip bands caused by the propagation of dislocations. No evidence of nanoindentation-induced phase transformation or cracking patterns was observed up to the maximum load of $80 \mathrm{mN}$, from either XTEM or atomic force microscopy (AFM) of the mechanically deformed regions. Instead, XTEM revealed that the primary deformation mechanism in AlN thin films is via propagation of dislocations on both basal and pyramidal planes. Furthermore, the hardness and Young's modulus of AlN thin films estimated using the continuous contact stiffness measurements (CSMs) mode provided with the nanoindenter are 16.2 GPa and $243.5 \mathrm{GPa}$, respectively.
\end{abstract}

\section{Introduction}

AlN is a potential candidate for UV and deep-UV optical and high-power electronic devices because of its unique properties, such as wide bandgap $(6.2 \mathrm{eV})$, high-temperature stability, and excellent thermal conductivity $[1,2]$. However, in order to successfully implement the fabrication of AlNbased devices, a better understanding of the mechanical properties is of essential importance in addition to its optical and electrical performances. For instance, contact loading, which is frequently encountered during material processing, can significantly degrade the performance of the particular devices. Contact loading is a type of mechanical impact, and there are several issues to be addressed, especially for thin films that are ubiquitously used for various device configurations. First, the mechanical responses of a thin film to an applied load might be vastly different from those of the same material in bulk form. For this purpose, unfortunately, the traditional methods such as tensile measurements do not scale well into the micrometer and nanometer regions. Secondly, the role of structural changes under contact loading is largely underestimated because of the difficulties in directly probing the structural properties of materials affected by the contact interaction. Therefore, methods capable of evaluating the mechanical properties of thin films in more precise fashion are in order. The nanoindentation technique developed some decades ago is useful and widely employed to measure the mechanical properties of thin films [3-6] and/or local structure of various materials down to submicro- or nanoscales [7-9].

In this study the mechanical characteristics of AlN thin films deposited on $c$-plane sapphire substrates using a helicon sputtering system were systematically investigated by combining the load-displacement data obtained from nanoindentation experiments with the microstructure observations performed in the vicinity of the indents by using atomic force microscopy (AFM) and cross-sectional transmission electron microscopy (XTEM). The important aspects of the underlying deformation mechanism governing the contact-induced deformation of AlN thin films with different films microstructures were revealed. The results obtained in this study should have technological implications for assessing the robustness against the usual fabrication processes of making AlN-based devices. 


\section{Experimental Details}

Experimentally, $1 \mu \mathrm{m}$ thick AlN thin films were deposited on $c$-plane sapphire substrates by using the helicon sputtering system. The detailed growth procedures of the present AlN/csapphire films were similar to those practiced previously to deposit AlN films on $\operatorname{Si}(111)$ substrates and can be found elsewhere [10]. The XRD results and XTEM images confirmed the high-quality hexagonal wurtzite structure of the current AlN/c-sapphire films in our previous study [11]. The AlN/c-sapphire films in the present study have more epitaxial characteristics as compared to the columnar grain structure obtained in AlN/Si(111) films [10]. The different film structures presumably are the result of the difference in the extent of film-substrate lattice mismatch. This, as will be discussed below, has led to quite different indentationinduced deformation behaviors.

The nanomechanical properties of AlN thin films were characterized using a nanoindentation technique with a diamond Berkovich indenter tip. The indentation measurement incorporated additional harmonic modulations with amplitude of $2 \mathrm{~nm}$ and a frequency of $45 \mathrm{~Hz}$ following a continuous stiffness (CSM) technique [12], which was accomplished by superimposing a small oscillation on the primary loading signal and analyzing the resulting response of the system by using a lock-in amplifier. Prior to real measurement, the indenter was loaded and unloaded three times to ensure that the tip was properly in contact with the surface of AlN thin films and that any parasitical phenomenon is released from the measurement. At the fourth time, the indenter was loaded at a strain rate of $0.05 \mathrm{~s}^{-1}$ until reaching an indent depth of $80 \mathrm{~nm}$ and was held for $10 \mathrm{sec}$. Then, it was withdrawn with the same strain rate until $10 \%$ of the peak load was reached. The load-displacement measurements were used to compute mechanical properties of the specimen. Each of the tests was performed until the thermal drift had dropped below $0.01 \mathrm{~nm} / \mathrm{s}$. At least ten indents were conducted on each sample with the adjacent two indents being separated by at least $10 \mu \mathrm{m}$ to avoid mutual interaction. In addition, it is generally accepted that the indentation depth should never exceed $30 \%$ of the sample thickness to avoid the substrate effect on hardness and modulus measurements [13]. Our samples and test methodology were satisfactory based on this criterion.

Furthermore, to reveal the role played by the nucleation and propagation of dislocations during indentationinduced deformation, cyclic nanoindentation tests were also performed in this study. These tests were carried out by the following sequence. First, the indenter was loaded to some chosen load and then unloaded by $90 \%$ of the previous load, which completed the first cycle. It then was reloaded to a larger chosen load and unloaded by $90 \%$ for the second cycle. Figure 1 illustrates a typical cyclic indentation test repeated for 5 cycles. Note that, in each cycle, the indenter was held for $30 \mathrm{~s}$ at $10 \%$ of its previous maximum load for thermal drift correction and for assuring that complete unloading was achieved. The thermal drift was kept below $\pm 0.05 \mathrm{~nm} / \mathrm{s}$ for all indentations considered in this study. The same loading/ unloading rate of $10 \mathrm{mN} / \mathrm{s}$ was used.

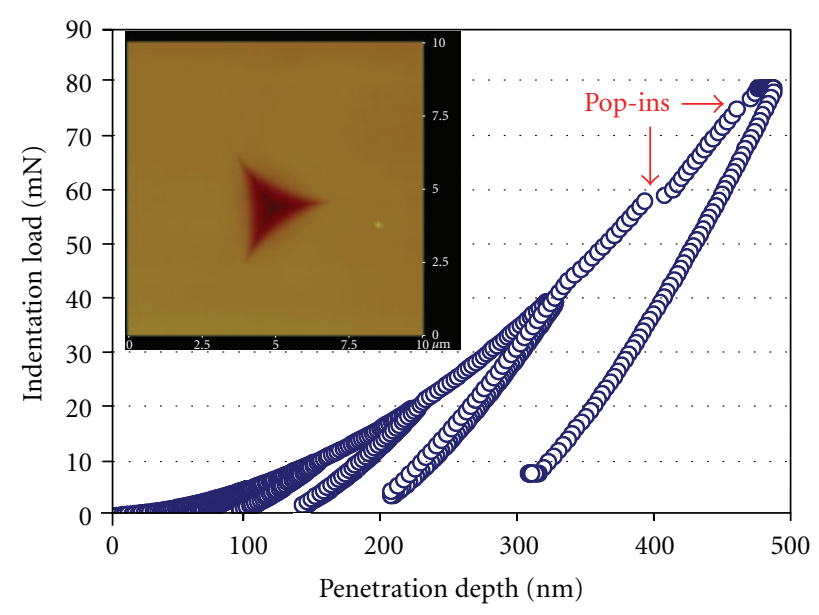

FIGURE 1: Typical cyclic nanoindentation load-displacement curves for AlN thin film obtained with a Berkovich indenter. Results show clear "pop-in" behaviors during loading, while no "pop-out" event is evident in unloading segments. AFM micrograph (inset) shows the indentation at an indentation load of $80 \mathrm{mN}$.

The XTEM samples were prepared from the indents by using a dual-beam-focused-ion-beam (FIB, Nova 220) station with $\mathrm{Ga}$ ions at $30 \mathrm{keV}$. Prior to milling, a Pt layer of $\sim 1 \mu \mathrm{m}$ thickness was deposited to protect the surface of the AlN films. The XTEM lamella was examined in a JEOL 2010F TEM operating at $200 \mathrm{kV}$ with a point-to-point resolution of $0.23 \mathrm{~nm}$ and a lattice resolution of $0.10 \mathrm{~nm}$.

\section{Results and Discussion}

A typical nanoindentation curve obtained for the $\mathrm{AlN} / \mathrm{c}$ sapphire films is displayed in Figure 2(a). The total penetration depth into thin film was about $80 \mathrm{~nm}$ with a peak load of $1.8 \mathrm{mN}$. With the continuous contact stiffness measurements, the penetration depth dependence of the hardness and Young's modulus was obtained from the loaddisplacement data by the analytic method developed by Oliver and Pharr [14], and the results are displayed in Figures 2(b) and 2(c), respectively. Clearly the indentationinduced deformation behaviors of the $\mathrm{AlN} / \mathrm{c}$-sapphire films are different from those observed in $\mathrm{AlN} / \mathrm{Si}(111)$ films [11] in several respects. First, in the present AlN/c-sapphire films, the first pop-in event occurs at a much smaller indentation load $(\sim 0.2 \mathrm{mN})$ as compared to $(\sim 2 \mathrm{mN})$ AlN/Si(111) films [11]. In the latter case, the pop-ins take place at a much higher loading due to the longitudinal shearing of the columnar grain boundaries which, in turn, induces arrays of shear steps at the film/substrate interface. Moreover, this behavior is also in contrast to that reported in [15], wherein no "pop-in" event was observed in AlN films deposited by filtered cathodic vacuum arc method on $\mathrm{Si}(100)$ substrates. The behaviors displayed in Figure 2(a), nevertheless, are more akin to those found in epitaxial GaN films grown on (0001) sapphire substrates [16], wherein active indentationinduced dislocation slip was responsible for the pop-ins taking place at relatively low loads. Furthermore, it is evident 


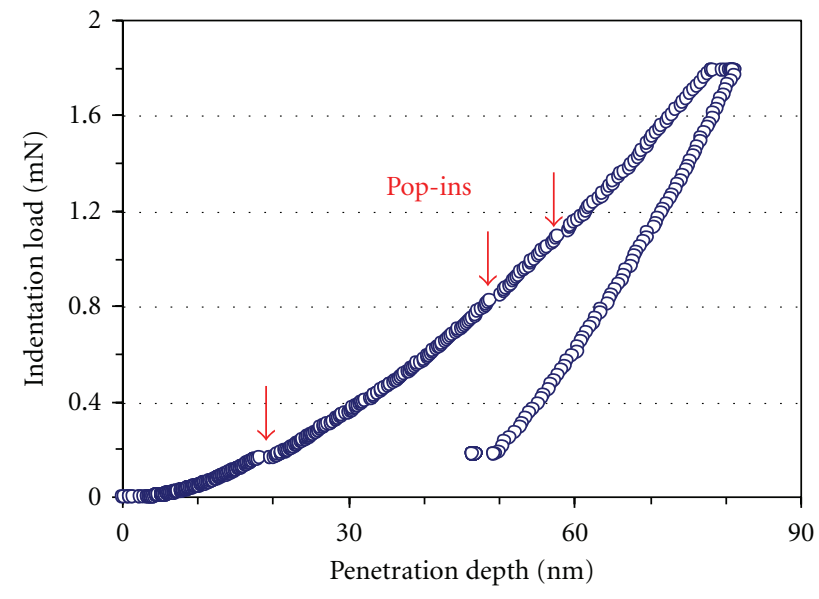

(a)

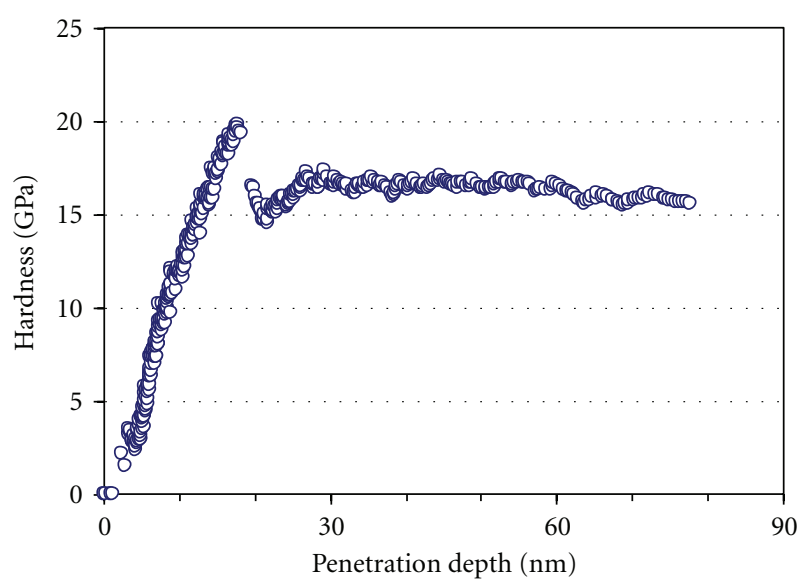

(b)

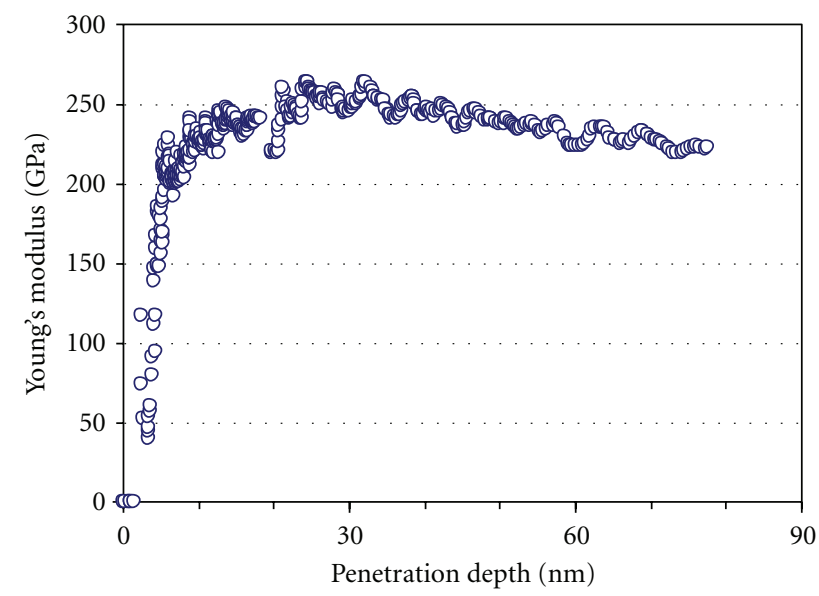

(c)

FIGURE 2: Nanoindentation results: (a) load-displacement curve of AlN thin film showing the "pop-in" behaviors during loading, (b) hardness-displacement curve, and (c) Young's modulus-displacement curve for AlN thin film.

TABLE 1: Hardness and Young's modulus of AlN thin films investigated in this study and those reported in the literatures.

\begin{tabular}{lccc}
\hline & AlN thin film & Hardness, $H(\mathrm{GPa})$ & Young's modulus, $E_{f}(\mathrm{GPa})$ \\
\hline Ji et al. [15] & AlN/Si(100) & $12.5 \sim 14.5$ & - \\
Barshilia et al. [16] & AlN/Si(100) & 12 & 225 \\
Yate et al. [17] & AlN/Si(100) & $10.56 \pm 0.07 \sim 17.2 \pm 0.6$ & $104 \pm 13 \sim 115 \pm 13$ \\
Laksana et al. [11] & AlN/Si(111) & $24.79 \pm 0.33$ & $223.92 \pm 5.34$ \\
Wang et al. [6] & AlN/GaN/c-sapphire & 22 & 332 \\
Jian and Juang\# & AlN/c-sapphire & 16.2 & 243.5 \\
\hline
\end{tabular}

${ }^{\#}$ The present study.

from Figure 2(b) that the first pop-in event coincides with the sudden drop in hardness at the penetration depth of $\sim 18 \mathrm{~nm}$. The hardness after the first pop-in for the present AlN/c-sapphire thin films remains nearly constant at 16.2 GPa with small fluctuations coinciding more or less with the subsequent multiple pop-ins over the entire penetration depth range, presumably associated with dislocation activity $[16,17]$. Similarly, as shown in Figure 2(c), Young's modulus of these films also displays a sudden drop occurring around the same penetration depth and then remains relatively constant at $243.5 \mathrm{GPa}$. Note that the curve of Young's modulus versus the penetration depth in the present films is qualitatively very different from that exhibited in columnar $\mathrm{AlN} / \mathrm{Si}(111)$ thin films [11], wherein Young's modulus continuously decreases with the penetration depth. Interestingly, the behavior is again very similar to that exhibited in the hexagonal GaN thin films grown on (0001)sapphire substrate [18]. Furthermore, the relevant literatures 
of mechanical properties (hardness and Young's modulus) of AlN thin films are listed in Table 1.

Although the resolution was much reduced due to larger indentation load, similar pop-ins phenomena are also evident in Figure 1, where the load-displacement results were obtained with a much larger load of $80 \mathrm{mN}$ and deeper penetration up to $500 \mathrm{~nm}$. The fact that pop-ins phenomena are observable over such a wide range of indentation load and penetration depth indicates the intimate relations between the pop-in events and the plastic deformation of the film. Furthermore, since the pop-ins phenomena are randomly distributed on the loading curve, as seen in Figure 1 and Figure 2(a), and each curve is associated with a different stress rate that increases with the maximum indentation load, it is suggested that the first pop-in is not thermally activated. Instead, as mentioned above, these phenomena occur in a wide variety of materials [18-20] and are attributed to dislocation nucleation and propagation during loading, or microcracking [21]. Also, the reverse discontinuities in the unloading segment of the load-displacement curve, the so-called "pop-out" event, commonly observed in $\mathrm{Si}$, which is attributed to pressure-induced phase transition [22] are not observed here as well. Therefore, the first pop-in event may reflect the transition from perfectly elastic to plastic deformation, that is, it is the onset of plasticity in AlN thin film. The corresponding shear stress under the Berkovich indenter at a critical indentation load $P_{\text {critical }}$, where the loaddisplacement discontinuity occurs, can be determined by using the following relation [23]:

$$
\tau_{\max }=0.31\left(\frac{6 P_{\text {critical }} E^{2}}{\pi^{3} R^{2}}\right)^{0.33},
$$

where $R$ is the radius of the tip of the indenter and $E$ is defined in terms of Young's moduli and Poisson's ratios of the diamond indenter and thin film. The maximum shear stress, $\tau_{\text {max }}$, of the present AlN thin film is $5.4 \mathrm{GPa}$ as compared to the value of $25.4 \mathrm{GPa}$ for the columnar AlN films grown on $\mathrm{Si}(111)$ substrates, where grain boundaries may inhibit longer range dislocation movements.

The multiple pop-ins displayed by the AlN thin films are similar to phenomena previously seen in hexagonal structured single-crystal $\mathrm{ZnO}$ [24], GaN thin films [25], and GaN/AlN multilayers [26] and attributed to dislocation nucleation, propagation, and pile-up or basal planes. In contrast, materials with cubic structure such as InP and GaAs normally exhibit only single pop-in characteristics [27]. Thus, the above observations suggest that multiple pop-ins phenomena are indeed specific to materials with the hexagonal lattice structure. Furthermore, the AFM observation shown in the inset of Figure 1 does not reveal any evidence of material pile-ups or signs of crack formation on the surface of the AlN/c-sapphire films around the indented area. Since there are no "pop-outs" phenomena on the unloading segment (Figure 1) it is suggested that phase transitions like that observed in indented $\mathrm{Si}$ [22] probably are not occurring in the present case. A pressure-induced phase transformation has been theoretically predicted at $16.6 \mathrm{GPa}$ for intrinsic AlN [28]. In fact, it was reported experimentally

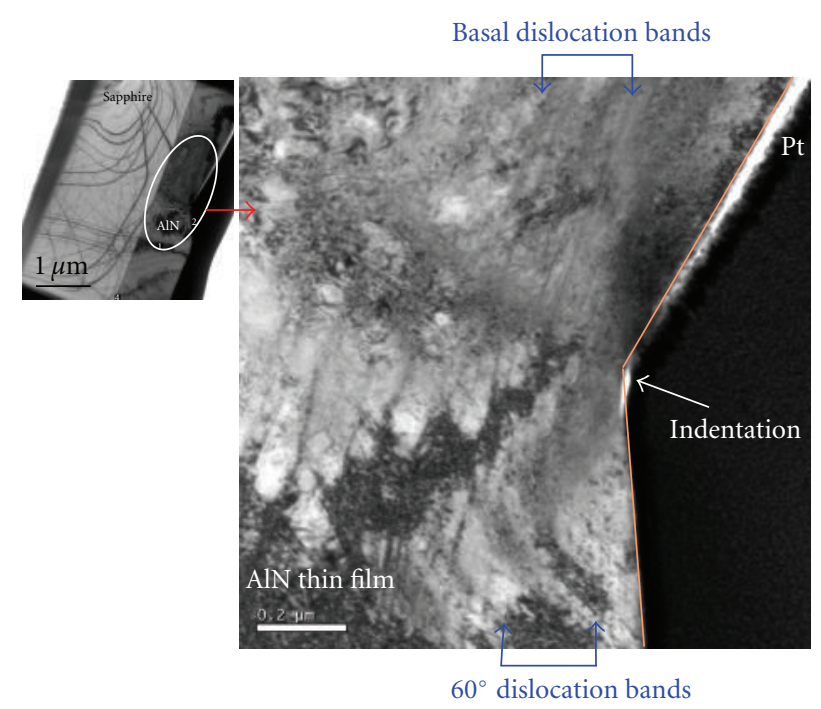

FIGURE 3: A bright-field XTEM image immediately under the Berkovich indent applied on the AlN thin film with an indentation load of $80 \mathrm{mN}$.

that AlN undergoes a wurtzite-to-rocksalt phase transition when subjected to a stress around $22.9 \mathrm{GPa}$ [29] in a diamond anvil cell experiment at room temperature. These values are only slightly higher than the contact pressure (i.e., the hardness) achieved in this present work, yet no indication of phase transition was detected. Consequently, the present results strongly suggest that nanoindentationinduced deformation in these AlN/c-sapphire films is predominantly due to dislocation nucleation and propagation. This plastic deformation process is complicated, and more detailed studies with the aid of XTEM techniques will be necessary to delineate what happens to the material as the indenter penetrates into the surface.

Figure 3 shows a bright-field XTEM image of an AlN $/ c$ sapphire thin film after indentation at a load of $80 \mathrm{mN}$. It clearly shows that the film is highly epitaxial with no feature of columnar structure. Moreover, it also shows that, within the entire film, the deformation features underneath the indented spot are primarily manifested by dislocation activities, namely, slip bands aligned parallel to the $\{0001\}$ basal planes. In addition, slip bands oriented at $\sim 60^{\circ}$ to the sample surface are also found. The $\sim 60^{\circ}$ slip bands, which are believed to originate from dislocations gliding along the $\{10 \overline{1} 1\}$ pyramidal planes, however, are distributing in much shallower regions near the contacting surface. This indicates that a much higher stress level is needed to activate these slip systems as compared to the ones along the basal planes. The distorted slip bands and the extremely high dislocation densities at the intersections indicate highly strained state of the material. Nevertheless, even at the submicron scale, no evidence of subsurface cracking and thin film fragmentation was observed. Since the multiple pop-ins phenomena are usually observed after permanent plastic deformation has occurred, two possible mechanisms, 
namely, deformation-induced phase transition [22, 30-32] and fracture of the thin films [33], can basically be ruled out. The most likely mechanism responsible for the multiple popins appears to be associated with the activation of dislocation sources [34]. In this scenario, plastic deformation prior to the pop-in event is associated with the individual movement of a small number of newly nucleated and also preexisting dislocations. As the number of dislocations is increased and entangled with each other providing dislocations pinning, large shear stress is quickly accumulated underneath the indenter tip. When the local stress reaches some threshold level, a burst of collective dislocation movement on the easy slip systems is activated, leading to a large release of local stress and a pop-in event on the load-displacement curve.

Consequently, in the current study, there are, at least, three possible mechanisms that can be responsible for this behavior. First, the specific hexagonal lattice structure of AlN offers the possibility of interactions and pinning between dislocations gliding along the pyramidal and basal planes. Second, the usual "slip-stick" behavior [25] can also prevail by the interactions between the as-grown dislocations and those being punched out by nanoindentation loading. Finally, the behavior could just be a consequence of punching out of the threading dislocations above a critical threshold energy by nanoindentation. That is, the threading dislocations originally existing in the AlN thin films could respectively display sudden propagation when they acquire enough energy from the deformation, resulting in the pop-ins behaviors. The fact that multiple pop-ins phenomena are observed only in hexagonal structured materials strongly suggests the first mechanism might be of primary importance.

\section{Conclusion}

In summary, the Berkovich nanoindentation, FIB, and TEM techniques were used to investigate the mechanical deformation behaviors of AlN thin films deposited on $c$-sapphire substrates by helicon sputter deposition. Multiple pop-ins phenomena were found in the load-displacement curve on loading, while no pop-out was observed during unloading cycle. The deformation behaviors of the current epitaxial AlN/c-sapphire thin films were in sharp contrast to the columnar-structured AlN/Si(111) thin films reported previously, indicating the prominent role played by the nanoscale grain boundaries in inhibiting the dislocation movements in the latter case. According to the XTEM observations, the nanoindentation-induced mechanical deformation is due primarily to the generation and propagation of dislocations gliding along the pyramidal and basal planes specific to the hexagonal structure of AlN rather than by indentationinduced phase transformations. In addition, the hardness and Young's modulus of the AlN/sapphire films determined by the continuous stiffness measurement (CSM) technique are $16.2 \mathrm{GPa}$ and $243.5 \mathrm{GPa}$, compared to $24.8 \mathrm{GPa}$ and 223.9 GPa, respectively, for AlN/Si(111) films. The apparent difference in hardness is attributed to the film microstructure obtained when different substrates are used.

\section{Acknowledgments}

This work was partially supported by the National Science Council of Taiwan, under Grant no. NSC100-2221-E-214024. J. Y. Juang is partially supported by the National Science Council and the MOE-ATU program operated at NCTU. The authors like to thank Dr. Y.-S. Lai, Dr. P.-F. Yang, and Professor H.-L. Kao for their technical supports.

\section{References}

[1] S. T. Strite and H. Morkoç, "GaN, AlN, and InN: a review," Journal of Vacuum Science and Technology, vol. 10, pp. 12371266, 1992.

[2] S. Nakamura, T. Mukai, and M. Senoh, "Candela-class highbrightness InGaN/AlGaN double-heterostructure blue-lightemitting diodes," Applied Physics Letters, vol. 64, no. 13, pp. 1687-1689, 1994.

[3] S.-R. Jian, J. S.-C. Jang, Y.-S. Lai et al., "Mechanical properties of InGaN thin films deposited by metal-organic chemical vapor deposition," Materials Chemistry and Physics, vol. 109, no. 2-3, pp. 360-364, 2008.

[4] S. R. Jian, G. J. Chen, J. S. C. Jang, and Y. Y. Liao, "Nanomechanical properties of FePtPd ternary alloy thin films," Thin Solid Films, vol. 517, no. 17, pp. 4883-4887, 2009.

[5] S. R. Jian, G. J. Chen, and T. C. Lin, "Berkovich nanoindentation on AlN thin films," Nanoscale Research Letters, vol. 5, no. 6, pp. 935-940, 2010.

[6] S.-K. Wang, T.-C. Lin, S.-R. Jian, J.-Y. Juang, J. S.-C. Jang, and J.-Y. Tseng, "Effects of post-annealing on the structural and nanomechanical properties of $\mathrm{Ga}$-doped $\mathrm{ZnO}$ thin films deposited on glass substrate by rf-magnetron sputtering," Applied Surface Science, vol. 258, no. 3, pp. 1261-1266, 2011.

[7] L. Liu, G. Cao, and X. Chen, "Mechanisms of nanoindentation on multiwalled carbon nanotube and nanotube cluster," Journal of Nanomaterials, vol. 2008, Article ID 271763, 12 pages, 2008.

[8] L. Bao, Z.-H. Xu, R. Li, and X. Li, "Catalyst-free synthesis and structural and mechanical characterization of single crystalline $\mathrm{Ca}_{2} \mathrm{~B}_{2} \mathrm{O}_{5} \cdot \mathrm{H}_{2} \mathrm{O}$ nanobelts and stacking faulted $\mathrm{Ca}_{2} \mathrm{~B}_{2} \mathrm{O}_{5}$ nanogrooves," Nano Letters, vol. 10, no. 1, pp. 255-262, 2010.

[9] T. H. Sung, J. C. Huang, J. H. Hsu, and S. R. Jian, "Mechanical response of GaN film and micropillar under nanoindentation and microcompression," Applied Physics Letters, vol. 97, no. 17, Article ID 171904, 2010.

[10] S.-R. Jian, G.-J. Chen, H.-G. Chen et al., "Cross-sectional transmission electron microscopy studies for deformation behaviors of AlN thin films under Berkovich nanoindentation," Journal of Alloys and Compounds, vol. 504, supplement 1, pp. S395-S398, 2010.

[11] C. P. Laksana, M.-R. Chen, Y. Liang et al., "Deep-UVsensors based on SAW oscillators using low-temperature-grown AlN films on sapphires," IEEE Transactions on Ultrasonics, Ferroelectrics, and Frequency Control, vol. 58, no. 8, pp. 1688-1693, 2011.

[12] X. Li and B. Bhushan, "A review of nanoindentation continuous stiffness measurement technique and its applications," Materials Characterization, vol. 48, no. 1, pp. 11-36, 2002.

[13] X. Li, H. Gao, C. J. Murphy, and K. K. Caswell, "Nanoindentation of Silver Nanowires," Nano Letters, vol. 3, no. 11, pp. 1495-1498, 2003.

[14] W. C. Oliver and G. M. Pharr, "Improved technique for determining hardness and elastic modulus using load and 
displacement sensing indentation experiments," Journal of Materials Research, vol. 7, no. 6, pp. 1564-1580, 1992.

[15] X. H. Ji, S. P. Lau, G. Q. Yu, W. H. Zhong, and B. K. Tay, "Structural properties and nanoindentation of AlN films by a filtered cathodic vacuum arc at low temperature," Journal of Physics D, vol. 37, no. 10, pp. 1472-1477, 2004.

[16] H. C. Barshilia, B. Deepthi, and K. S. Rajam, "Growth and characterization of aluminum nitride coatings prepared by pulsed-direct current reactive unbalanced magnetron sputtering," Thin Solid Films, vol. 516, no. 12, pp. 4168-4174, 2008.

[17] L. Yate, J. C. Caicedo, A. H. Macias et al., "Composition and mechanical properties of $\mathrm{AlC}, \mathrm{AlN}$ and $\mathrm{AlCN}$ thin films obtained by r.f. magnetron sputtering," Surface and Coatings Technology, vol. 203, no. 13, pp. 1904-1907, 2009.

[18] C. H. Tsai, S. R. Jian, and J. Y. Juang, "Berkovich nanoindentation and deformation mechanisms in GaN thin films," Applied Surface Science, vol. 254, no. 7, pp. 1997-2002, 2008.

[19] J. E. Bradby, J. S. Williams, and M. V. Swain, "Pop-in events induced by spherical indentation in compound semiconductors," Journal of Materials Research, vol. 19, no. 1, pp. 380-386, 2004.

[20] A. J. Haq, P. R. Munroe, M. Hoffman, P. J. Martin, and A. Bendavid, "Nanoindentation-induced deformation behaviour of diamond-like carbon coatings on silicon substrates," Thin Solid Films, vol. 515, no. 3, pp. 1000-1004, 2006.

[21] S. J. Bull, "Nanoindentation of coatings," Journal of Physics D, vol. 38, no. 24, pp. R393-R413, 2005.

[22] S.-R. Jian, G.-J. Chen, and J.-Y. Juang, "Nanoindentationinduced phase transformation in ( $\left.\begin{array}{lll}1 & 1 & 0\end{array}\right)$-oriented Si singlecrystals," Current Opinion in Solid State and Materials Science, vol. 14, no. 3-4, pp. 69-74, 2010.

[23] K. L. Johnson, Contact Mechanics, Cambridge University Press, Cambridge, UK, 1985.

[24] S. O. Kucheyev, J. E. Bradby, J. S. Williams, C. Jagadish, and M. V. Swain, "Mechanical deformation of single-crystal ZnO," Applied Physics Letters, vol. 80, no. 6, p. 956, 2002.

[25] J. E. Bradby, S. O. Kucheyev, J. S. Williams et al., "Indentationinduced damage in GaN epilayers," Applied Physics Letters, vol. 80, no. 3, p. 383, 2002.

[26] S.-R. Jian, J.-Y. Juang, N.-C. Chen, J. S.-C. Jang, J. C. Huang, and Y.-S. Lai, "Nanoindentation-induced structural deformation in GaN/AlN multilayers," Nanoscience and Nanotechnology Letters, vol. 2, no. 4, pp. 315-321, 2010.

[27] J. E. Bradby, J. S. Williams, J. Wong-Leung, M. V. Swain, and P. Munroe, "Mechanical deformation of InP and GaAs by spherical indentation," Applied Physics Letters, vol. 78, no. 21, pp. 3235-3237, 2001.

[28] I. Gorczyca, N. E. Christensen, P. Perlin, I. Grzegory, J. Jun, and M. Bockowski, "High pressure phase transition in aluminium nitride," Solid State Communications, vol. 79, no. 12, pp. 10331034, 1991.

[29] M. Ueno, A. Onodera, O. Shimomura, and K. Takemura, "Xray observation of the structural phase transition of aluminum nitride under high pressure," Physical Review B, vol. 45, no. 17, pp. 10123-10126, 1992.

[30] D. J. Oliver, J. E. Bradby, J. S. Williams, M. V. Swain, and P. Munroe, "Giant pop-ins and amorphization in germanium during indentation," Journal of Applied Physics, vol. 101, no. 4, Article ID 043524, 2007.

[31] D. J. Oliver, B. R. Lawn, R. F. Cook et al., "Giant pop-ins in nanoindented silicon and germanium caused by lateral cracking," Journal of Materials Research, vol. 23, no. 2, pp. 297301,2008 .
[32] D. J. Oliver, J. E. Bradby, S. Ruffell, J. S. Williams, and P. Munroe, "Nanoindentation-induced phase transformation in relaxed and unrelaxed ion-implanted amorphous germanium," Journal of Applied Physics, vol. 106, no. 9, Article ID 093509, 2009.

[33] X. Li and B. Bhushan, "Evaluation of fracture toughness of ultra-thin amorphous carbon coatings deposited by different deposition techniques," Thin Solid Films, vol. 355, pp. 330336, 1999.

[34] Y. Gaillard, C. Tromas, and J. Woirgard, "Pop-in phenomenon in $\mathrm{MgO}$ and LiF: observation of dislocation structures," Philosophical Magazine Letters, vol. 83, no. 9, pp. 553-561, 2003. 

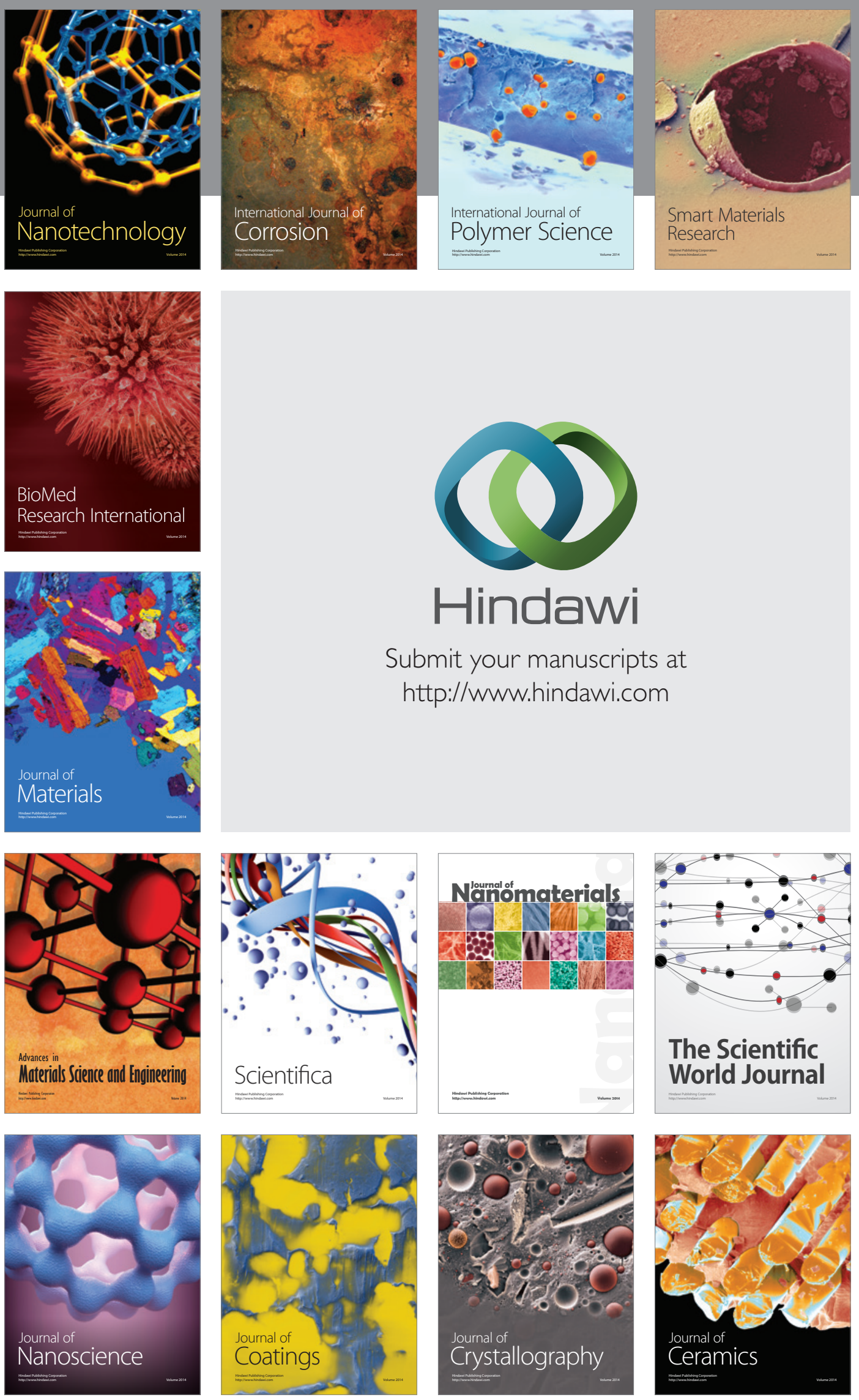

The Scientific World Journal

Submit your manuscripts at

http://www.hindawi.com

\section{World Journal}

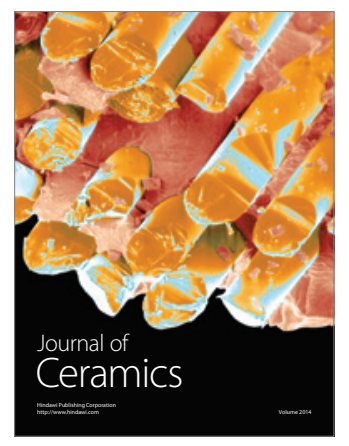

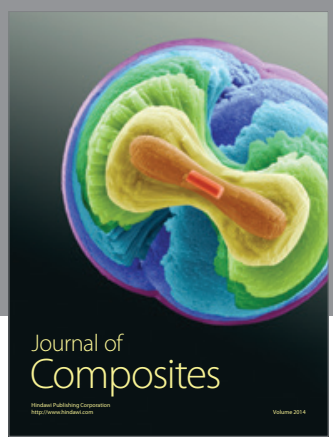
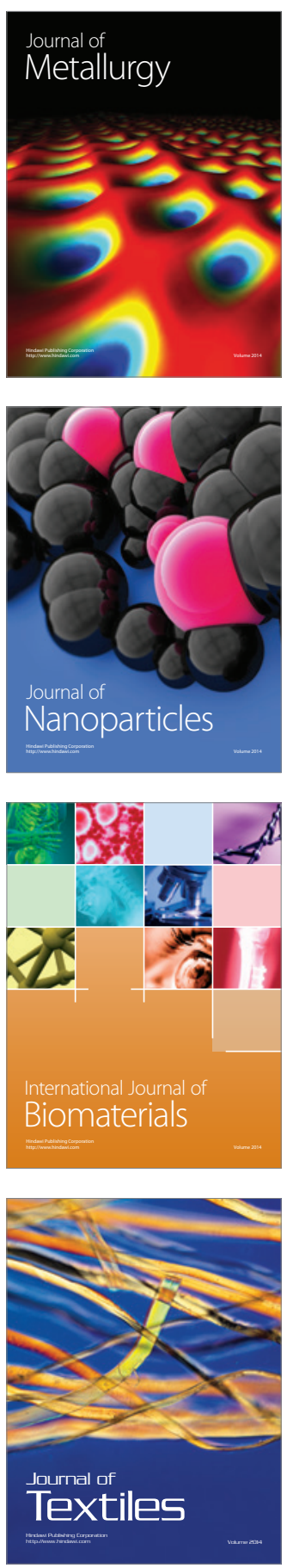